\title{
OSCILLATIONS OF HIGHER-ORDER NEUTRAL EQUATIONS
}

\author{
G. LADAS ${ }^{1}$ AND Y. G. SFICAS ${ }^{2}$
}

(Received 12 April 1985)

\begin{abstract}
Sufficient conditions are given for the occurrence of various types of asymptotic behaviour in the solution of a class of $n$th order neutral delay differential equations. The conditions are in the form of certain inequalities amongst the constants involved in the definition of the differential equations, and specify either oscillatory behavior, or asymptotic divergence, or solutions which converge to zero.
\end{abstract}

\section{Introduction}

A neutral delay differential equation (NDDE for short) is a differential equation in which the highest order derivative of the unknown function appears both with and without delays. Concerning existence, uniqueness and continuous dependence for NDDE the reader is referred to Driver [3] and [4], Bellman and Cooke [1] and Hale [6]. It is known that the behavior of solutions of NDDE exhibit features which are not true for plain delay differential equations. For example, Snow [11] has shown that even though the characteristic roots of a NDDE may all lie in the negative half-plane, it is still possible for the equation to have unbounded solutions.

In this paper we deal with the asymptotic and oscillatory behavior of the solutions of the NDDE of order $n \geqslant 1$,

$$
\frac{d^{n}}{d t^{n}}[y(t)+p y(t-\tau)]+q y(t-\sigma)=0, \quad t \geqslant t_{0}
$$

\footnotetext{
${ }^{1}$ Department of Mathematics, University of Rhode Island, Kingston, Rhode Island 02881, USA;

${ }^{2}$ Department of Mathematics, University of Ioannina, Ioannina 45332, Greece.

(c) Copyright Australian Mathematical Society 1986, Serial-fee code 0334-2700/86
} 
where $q$ is a positive constant, the delays $\tau$ and $\sigma$ are nonnegative, and the coefficient $p$ is a real parameter. The case $n=1$ has recently been studied by Ladas and Sficas [7] and by Grammatikopoulos, Grove and Ladas [5].

From the point of view of applications, NDDE appear as models of electrical networks which contain lossless transmission lines. Such networks arise, for example, in high speed computers where lossless transmission lines are used to interconnect switching circuits. See [2] and [10].

As usual, a solution of Equation (1) is called oscillatory if it has arbitrarily large zeros and nonoscillatory if it is eventually positive or negative.

\section{Asymptotic behavior of nonoscillatory solutions}

In this section we will study the asymptotic behavior of the nonoscillatory solutions of the $n$th order NDDE

$$
\frac{d^{n}}{d t^{n}}[y(t)+p y(t-\tau)]+q y(t-\sigma)=0, \quad t \geqslant t_{0},
$$

under the hypothesis:

(H) $q$ is a positive constant, $p$ is a real number, and $\tau$ and $\sigma$ are nonnegative constants.

The main feature of our results is that for $n$ odd, the point $p=-1$ is a bifurcation point for the asymptotic nature of the nonoscillatory solutions of Equation (1). However, this is not true, in general for even $n$. More precisely, the following result is true.

THEOREM 1. Consider the NDDE (1) and assume that the hypothesis (H) is satisfied. Then, the following statements are true.

(a) Assume that $n$ is odd and that $p<-1$. Then every nonoscillatory solution of Equation (1) tends to $+\infty$ or $-\infty$ as $t \rightarrow \infty$.

(b) Assume that $n$ is odd or even and that $p>-1$. Then every nonoscillatory solution of Equation (1) tends to zero as $t \rightarrow \infty$.

Proof. (a) As the negative of a solution of Equation (1) is also a solution of the same equation, it suffices to prove that an eventually positive solution of Equation (1) tends to $+\infty$ as $t \rightarrow \infty$. For our convenience we will adopt the convention that all inequalities about values of functions are assumed to hold eventually for all $t$ sufficiently large. Set

$$
z(t)=y(t)+p y(t-\tau)
$$

Then

$$
z^{(n)}(t)=-q y(t-\sigma)<0
$$


and so $z^{(n-1)}(t)$ is (eventually) strictly decreasing. Also all the derivatives of $z$ of order less than or equal to $n-1$ are monotonic functions. From (3) it follows that either

$$
\lim _{t \rightarrow \infty} z^{(n-1)}(t)=-\infty
$$

or

$$
L \equiv \lim _{t \rightarrow \infty} z^{(n-1)}(t) \text { is finite. }
$$

However, we claim that (5) is impossible. Otherwise, integrating (3) from $t_{1}$ to $t$, with $t_{1}$ sufficiently large, and letting $t \rightarrow \infty$ we find

$$
L-z^{(n-1)}\left(t_{1}\right)=-q \int_{t_{1}}^{\infty} y(s-\sigma) d s
$$

which implies that $y \in L^{1}\left[t_{1}, \infty\right)$. Thus, from (2), $z \in L^{1}\left[t_{1}, \infty\right)$ and since $z$ is monotonic, it follows that

$$
\lim _{t \rightarrow \infty} z(t)=0
$$

and so $L=0$. As the function $z^{(n-1)}(t)$ decreases to zero, it follows that

$$
z^{(n-1)}(t)>0 \text {. }
$$

Also (6) implies that consecutive derivatives of $z$ must alternate sign. Thus, in view of (7) and the fact that $n$ is odd, we have

$$
z(t)>0 \text {. }
$$

However, (8) implies that

$$
y(t)>-p y(t-\tau)
$$

and by iteration

$$
y(t+k \tau)>(-p)^{k} y(t) \rightarrow \infty \text { as } k \rightarrow \infty
$$

which contradicts the fact that $y \in L^{1}\left[t_{1}, \infty\right)$ and establishes the claim that (4) holds. Furthermore, (4) implies that

$$
\lim _{t \rightarrow \infty} z(t)=-\infty
$$

But

$$
p y(t-\tau)<y(t)+p y(t-\tau)=z(t) \rightarrow-\infty \quad \text { as } t \rightarrow \infty
$$

and since $p<0$ it follows that

$$
\lim _{t \rightarrow \infty} y(t)=\infty
$$

The proof of (a) is complete.

(b) Let $y(t)$ be an eventually positive solution of Equation (1). Introducing the function $z$, as in (2) of the proof of part (a), we find that either (4) or (5) holds. 
We claim that, in this case, (4) is impossible. Otherwise, we shall also have that

$$
\lim _{t \rightarrow \infty} z(t)=-\infty
$$

which implies that

$$
0<y(t)<-p y(t-\tau)
$$

and by iteration

$$
0<y(t+k \tau)<(-p)^{k} y(t) .
$$

We have $p>-1$ and so (10) is impossible for $p \geqslant 0$ while (11) implies that $\lim _{t \rightarrow \infty} y(t)=0$ for $-1<p<0$. But in the latter case (9) is impossible. We have established the validity of (5) for any $p>-1$. We also have, in this case, as in the proof of (a), that (6) and (7) hold, the limit $L$ in (5) is 0 , and that consecutive derivatives of $z$ must alternate sign.

Next, we shall prove that $\lim _{t \rightarrow \infty} y(t)=0$. For $p \geqslant 0$ this follows from the observation that

$$
0<y(t) \leqslant y(t)+p y(t-\tau)=z(t) \rightarrow 0 \text { as } t \rightarrow \infty .
$$

On the other hand, for $-1<p<0$ we shall apply Lemma 1(ii) of Ladas and Sficas [7] to the functions $f(t)=z(t)$ and $g(t)=y(t)$. According to this Lemma, if $f(t)=g(t)+p g(t-\tau)$ where $-1<p<0$, if $g$ is bounded and if the $\lim _{t \rightarrow \infty} f(t)$ exists, then the $\lim _{t \rightarrow \infty} g(t)$ also exists. Since the $\lim _{t \rightarrow \infty} z(t)=0$ it would then follow that $\lim _{t \rightarrow \infty} y(t)=0$. Therefore, to complete the proof of (b) it remains to show that for $-1<p<0$, the solution $y(t)$ is bounded. To this end, first we assume that $n$ is even. Then, from (7) and the fact that consecutive derivatives of $z(t)$ alternate sign, we find that $z(t)<0$. Hence

$$
y(t)<-p y(t-\tau)<y(t-\tau)
$$

which establishes the boundedness of $y(t)$. Next, assume that $n$ is odd. Assume, for the sake of contradiction that $y(t)$ is not bounded. Then, there exists a sequence of points $\left\{t_{k}\right\}$ such that $\lim _{k \rightarrow \infty} t_{k}=\infty, y\left(t_{k}\right)=\max _{s \leqslant t_{k}} y(s)$, and $\lim _{k \rightarrow \infty} y\left(t_{k}\right)=\infty$. We have

$$
z\left(t_{k}\right)=y\left(t_{k}\right)+p y\left(t_{k}-\tau\right) \geqslant y\left(t_{k}\right)+p y\left(t_{k}\right)=(1+p) y\left(t_{k}\right) .
$$

Hence

$$
\lim _{k \rightarrow \infty} z\left(t_{k}\right)=\infty
$$

which contradicts (6). The proof of the Theorem is complete.

REMARK 1 . When $n$ is even and $p<-1$, it is not true that the nonoscillatory solutions of Equation (1) tend to $\pm \infty$ as $t \rightarrow \infty$ (as in case (a) of Theorem 1) and it is not true that they tend to zero either. This follows from the NDDE

$$
d^{2} / d t^{2}[y(t)-2 y(t-\log 2)]+(3 / e) y(t-1)=0
$$


which has the nonoscillatory solution $y(t)=e^{-t}$ and from the NDDE

$$
d^{2} / d t^{2}[y(t)-4 y(t-\log 2)]+e y(t-1)=0
$$

which has the nonoscillatory solutions $\pm e^{t}$.

REMARK 2. When $n$ is odd and $p=-1$, we shall prove in the next section that all solutions of Equation (1) oscillate. However, this is not true for $n$ even and $p=-1$. Indeed, the NDDE

$$
d^{2} / d t^{2}[y(t)-y(t-\log 2)]+(1 / e) y(t-1)=0
$$

has the nonoscillatory solution $y(t)=e^{-t}$.

REMARK 3. When $n$ is even and $p=-1$, we can show that every nonoscillatory solution of Equation (1) is bounded. Indeed, if $y(t)$ is eventually positive, then as in the proof of Theorem 1, it follows that $z(t)<0$. That is, $y(t)<y(t-\tau)$ which proves our claim. However, in agreement with the example in Remark 2, we make the following conjecture.

Conjecture. Assume that $n$ is even and $p=-1$. Then every nonoscillatory solution of Equation (1) tends to zero as $t \rightarrow \infty$.

We close this section by analyzing a little more the asymptotic behavior of the nonoscillatory solutions of Equation (1) when $n$ is even and $p<-1$. Let $y(t)$ be an eventually positive solution of Equation (1) and set $z(t)=y(t)+p y(t-\tau)$. Then, by arguments similar to those in the proof of Theorem 1, it follows that either

$$
\lim _{t \rightarrow \infty} z^{(i)}(t)=-\infty, \quad i=0,1,2, \ldots, n-1
$$

or

and

$$
z(t)<0, z^{\prime}(t)>0, \quad z^{\prime \prime}(t)<0, \ldots, z^{(n-1)}(t)>0
$$

$$
\lim _{t \rightarrow \infty} z^{(i)}(t)=0, \quad i=0,1,2, \ldots, n-1 .
$$

When (12) holds,

and so

$$
p y(t-\tau)<z(t) \rightarrow-\infty \quad \text { as } t \rightarrow \infty
$$

$$
\lim _{t \rightarrow \infty} y(t)=+\infty
$$

When (13) holds we make the following conjecture.

Conjecture. Assume $n$ is even, $p<-1$ and that (13) holds for an eventually positive solution of Equation (1). Then

$$
\lim _{t \rightarrow \infty} y(t)=0 \text {. }
$$




\section{Sufficient conditions for oscillation}

Here we shall obtain sufficient conditions for the oscillation of solutions of Equation (1). First, we should collect some facts that were established within the proof of Theorem 1 for $p \neq-1$ and which for $p=-1$ follow easily by arguments similar to those in the proof of Theorem 1. As in proof of Theorem 1, we assume that $y(t)$ is an eventually positive solution of Equation (1). Set

$$
z(t)=y(t)+p y(t-\tau) .
$$

Then, for any $i=0,1,2, \ldots, n-1$, we have

$$
\lim _{t \rightarrow \infty} z^{(t)}(t)=\left\{\begin{array}{l}
-\infty \text { if } p<-1 \text { and } n \text { is odd, } \\
0 \text { if } p \geqslant-1
\end{array}\right.
$$

For $p=-1$, the above result follows by an argument similar to that in the proof of Theorem 1. Also, for $p \geqslant-1$ (and any $n$, even or odd) we have that

$$
z^{(n-1)}(t)>0
$$

and

$$
z^{(t)}(t) z^{(t+1)}(t)<0 \text { for } i=0,1,2, \ldots, n-1 .
$$

From (14) and (15) we find the following result which for easy reference we state as a lemma.

Lemma 1. Assume $p \geqslant-1$. Then $z(t)$ is bounded. Furthermore, $z(t)>0$ for $n$ odd while $z(t)<0$ for $n$ even.

It is useful to note that the function $z(t)$ as defined above is an $n$-times continuously differentiable solution of Equation (1). That is,

$$
z^{(n)}(t)+p z^{(n)}(t-\tau)+q z(t-\sigma)=0 .
$$

Set

$$
w(t)=z(t)+p z(t-\tau) .
$$

Then, from (16), it follows that

$$
w^{(n)}(t)=-q z(t-\sigma) .
$$

Furthermore, $w$ is itself a $2 n$ - times continuously differentiable solution of Equation (1). That is,

$$
w^{(n)}(t)+p w^{(n)}(t-\tau)+q w(t-\sigma)=0 .
$$

From (17), (12) and (13) it follows that, for $i=0,1,2, \ldots, n$

$$
\lim _{t \rightarrow \infty} w^{(t)}(t)=\left\{\begin{array}{l}
+\infty, \quad \text { if } p<-1 \text { and } n \text { is odd, } \\
0, \text { if } p \geqslant-1 .
\end{array}\right.
$$


Moreover, for $p \geqslant-1$, consecutive derivatives of $w$ have alternate signs. For future reference we state the following lemma about $w$.

Lemma 2. Assume $n$ is odd. Then, for any $p \in(-\infty,+\infty)$,

$$
w^{(n+1)}(t)>0 \text { and } w(t)>0 .
$$

The following lemma, which will be essential in the proofs of our oscillation theorems, has been extracted from Ladas and Stavroulakis [8] and [9].

LEMMA 3. Assume that $r$ and $\mu$ are positive constants and suppose that

$$
r^{1 / n}(\mu / n)>1 / e .
$$

Then

(i) for $n$ odd, the inequality

$$
x^{(n)}(t)-r x(t+\mu) \geqslant 0
$$

has no eventually positive solution;

(ii) for $n$ odd, the inequality

$$
x^{(n)}(t)+r x(t-\mu) \leqslant 0
$$

has no eventually positive solution;

(iii) for $n$ even, the inequality

$$
x^{(n)}(t)-r x(t-\mu) \leqslant 0
$$

has no eventually negative bounded solution;

(iv) for $n$ even, the inequality

$$
x^{(n)}(t)-r x(t+\mu) \leqslant 0
$$

has no eventually negative unbounded solution.

We now are ready to state several sufficient conditions for the oscillation of solutions of the NDDE

$$
\frac{d^{n}}{d t^{n}}[y(t)+p y(t-\tau)]+q y(t-\sigma)=0, \quad t \geqslant t_{0},
$$

where the coefficients and the delays satisfy the hypothesis: $(\mathrm{H}) q$ is a positive constant, $p$ is a real number, and $\tau$ and $\sigma$ are nonnegative constants.

THEOREM 2. Assume $n$ is odd and that the hypothesis $(\mathrm{H})$ is satisfied. Then, each of the following three conditions implies that all solutions of Equation (1) oscillate.

$$
p<-1 \text { and }\left(-\frac{q}{1-p}\right)^{1 / n}(\tau-\sigma) / n>1 / e \text {; }
$$

$$
p=-1 \text {; }
$$

$$
p>-1 \text { and }\left(\frac{q}{1+p}\right)^{1 / n}(\sigma-\tau) / n>1 / e \text {. }
$$


Proof. Assume that one of the conditions (i)-(iii) is satisfied and that contrary to the conclusion of the theorem, Equation (1) has an eventually positive solution. Then, from Lemma $2, w^{(n+1)}(t)>0$ which implies that

$$
w^{(n)}(t)>w^{(n)}(t-\tau)
$$

and Equation (18) leads to the inequality

$$
(1+p) w^{(n)}(t-\tau)+q w(t-\sigma) \leqslant 0 .
$$

Also from Lemma 2,

$$
w(t)>0 .
$$

First, assume that (20) is satisfied. Then, from (23) we find that

$$
w^{(n)}(t)+\frac{q}{1+p} w(t+\tau-\sigma) \geqslant 0
$$

which by Lemma 3(i) has no eventually positive solution. This contradicts (24) and proves the Theorem when (20) is satisfied. Next, assume that (21) is satisfied. Then, from (23) we find that $w(t) \leqslant 0$ which contradicts (24). Finally, assume that (22) is satisfied. Then, from (23) we find that

$$
w^{(n)}(t)+\frac{q}{1+p} w(t-(\sigma-\tau)) \leqslant 0
$$

which by Lemma 3(ii) has no eventually positive solution. This contradicts (24) and completes the proof of Theorem 2.

Theorem 3. Assume $n$ is odd and that the hypothesis $(\mathrm{H})$ is satisfied. Suppose that $-1<p<0$, and

$$
q^{1 / n}(\sigma / n)>1 / e .
$$

Then every solution of Equation (1) oscillates.

Proof. Assume, for the sake of contradiction, that Equation (1) has an eventually positive solution $y(t)$. Set

$$
z(t)=y(t)+p y(t-\tau)
$$

Then

$$
z^{(n)}(t)=-q \nu(t-\tau)<0,
$$

and from Equation (16) we find the inequality

$$
z^{(n)}(t)+q z(t-\tau) \leqslant 0 .
$$

Also from Lemma 1

$$
z(t)>0
$$


But in view of Lemma 3(ii) and condition (25), Inequality (26) cannot have an eventually positive solution. This contradicts (27). The proof is complete.

Next, we turn to the case where $n$ is even.

TheOREM 4. Assume that $n$ is even and that the hypothesis $(\mathrm{H})$ is satisfied. Suppose

$$
p \geqslant 0 \text {. }
$$

Then every solution of Equation (1) oscillates.

Proof. Assume, for the sake of contradiction, that $y(t)$ is an eventually positive solution of Equation (1). Then, from (28),

$$
z(t)=y(t)+p y(t-\tau)>0,
$$

which contradicts Lemma 1 . The proof is complete.

TheOREM 5. Assume that $n$ is even and that the hypothesis $(\mathrm{H})$ is satisfied. Suppose that

$$
-1 \leqslant p<0
$$

and

$$
(-q / p)^{1 / n}(\sigma-\tau) / n>1 / e .
$$

Then every solution of Equation (1) oscillates.

Proof. Assume, for the sake of contradiction, that Equation (1) has an eventually positive solution $y(t)$. Then $z(t)=y(t)+p y(t-\tau)>p y(t-\tau)$ and so,

$$
y(t-\sigma)>\frac{1}{p} z(t-(\sigma-\tau)) .
$$

It follows that

$$
z^{(n)}(t)=-q y(t-\sigma)<-\frac{q}{p} z(t-(\sigma-\tau))
$$

or

$$
z^{(n)}(t)+\frac{p}{q} z(t-(\sigma-\tau))<0 .
$$

Also from Lemma 1, we have that

$$
z(t)<0 \text { and } z(t) \text { is bounded. }
$$

In view of (30) and Lemma 3(iii), it follows that Inequality (31) cannot have an eventually negative bounded solution. This contradicts (32) and completes the proof of the theorem. 
THEOREM 6. Assume that $n$ is even and that the hypothesis $(\mathrm{H})$ and the condition (30) are satisfied. Suppose that

$$
p<-1 \text {. }
$$

Then every bounded solution of Equation (1) oscillates.

Proof. Assume, for the sake of contradiction, that Equation (1) has an eventually positive and bounded solution $y(t)$. Then, as in the proof of Theorem 5 , (31) holds. Also, in view of the fact that $y(t)$ is bounded, (13) and in particular (32) is satisfied. As in the proof of Theorem 5, this leads to a contradiction. The proof is complete.

\section{References}

[1] R. Bellman and K. L. Cooke, Differential-difference equations (Academic Press, New York, 1963).

[2] R K. Brayton and R. A. Willoughby, "On the numerical integration of a symmetric system of difference-differential equations of neutral type", J. Math. Anal. Appl. 18 (1967), 182-189.

[3] R. D. Driver, "Existence and continuous dependence of solutions of a neutral functional-differential equation", Arch. Rational Mech. Anal. 19 (1965), 149-166.

[4] R. D. Driver, "A mixed neutral system", Nonlinear Anal.-TMA 8 (1984), 155-158.

[5] M. K. Grammatikopoulos, E. A. Grove and G. Ladas, "Oscillations of first order neutral delay differential equations" (to appear).

[6] J. Hale, Theory of functional differential equations (Springer-Verlag, New York, 1977).

[7] G. Ladas and Y. G. Sficas, "Oscillations of neutral delay differential equations" (to appear).

[8] G. Ladas and I. P. Stavroulakis, "On delay differential inequalities of higher order", Canad. Math. Bull. 25 (1982), 348-354.

[9] G. Ladas and I. P. Stavroulakis, "Oscillations of differential equations of mixed type", $J$. Math. Phys. Sci. (to appear).

[10] M. Slemrod and E. F. Infante, "Asymptotic stability criteria for linear systems of difference-differential equations on neutral type and their discrete analogues", J. Math. Anal. Appl. 38 (1972), 399-415.

[11] W. Snow, "Existence, uniqueness, and stability for nonlinear differential-difference equations in the neutral case", N.Y.U. Courant Inst. Math. Sci. Rep. IMM-NYU 328, (February 1965). 